\title{
Effect of sucrose fatty acid esters on the particle characteristics and flow properties of phytosterol nanodispersions
}

\begin{abstract}
The effect of four different types of sucrose fatty acid esters as nonionic emulsifiers on the physicochemical properties of water-soluble phytosterol nanodispersions was investigated. In general, the mean particle sizes of the prepared phytosterol nanodispersions ranged from 2.8 to $259.9 \mathrm{~nm}$. The phytosterol content in the final prepared nanodispersions ranged from 230.4 to $504.6 \mathrm{mg} / \mathrm{l}$. All of the prepared phytosterol nanodispersions exhibited pseudoplastic flow behavior, with low yield stress ranging from 0.630 to $9.183 \mathrm{mPa}$ and a low consistency coefficient of $0.608 і ̈ 88.710 \mathrm{mPas}$. Less than $1.5 \mathrm{\varepsilon l}$ of hexane residues per liter of prepared nanodispersions was found in the prepared phytosterol nanodispersions. Transmission electron microscopy (TEM) demonstrated that the prepared phytosterol nanoparticles were spherical in shape. In general, the sucrose fatty acid esters P-1570, L-1695 and S-1570 are appropriate for use in the preparation of phytosterol nanoparticles with small mean particle size at monomodal distribution with high clarity in appearance.
\end{abstract}

Keyword: Sucrose fatty acid esters; Phytosterol nanodispersions; Mean particle size; Flow properties; \% Transmittance 